\title{
LA EDUCACIÓN PARA EL DESARROLLO EN EL MARCO UNIVERSITARIO. ¿POR QUÉ Y PARA QUÉ?
}

\section{EDUCATION FOR DEVELOPMENT IN THE UNIVERSITY CONTEXT. WHY AND FOR WHAT?}

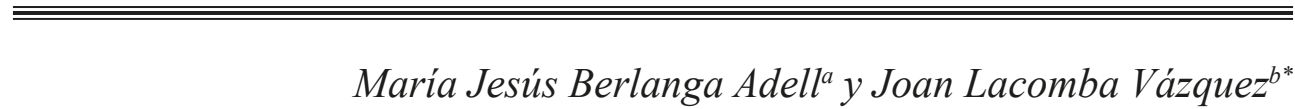

Fechas de recepción y aceptación: 26 de febrero de 2021 y 24 de marzo de 2021

DOI: https://doi.org/10.46583/edetania_2021.59.815

Resumen: La educación para el desarrollo se ha venido popularizando progresivamente en las universidades españolas en paralelo con la extensión de la cooperación para el desarrollo entre estas. La educación para el desarrollo se ha visto como una oportunidad para promover el compromiso social de la Universidad y fomentar valores de solidaridad y justicia a través de la formación universitaria. La educación para el desarrollo ha puesto de relieve el componente educativo de la Universidad, a menudo subsumido por el afán formativo. Sin embargo, el alcance de la educación para el desarrollo y, sobre todo, su transversalización en los programas de estudio universitarios siguen siendo muy limitados. El objetivo de este artículo es mostrar qué es y qué papel puede desempeñar la educación para el desarrollo en el contexto de la educación universitaria. Para ello recurrimos al análisis de fuentes secundarias, mediante la revisión tanto de bibliografía especializada como de literatura gris. Nuestra principal conclusión es que la educación para el desarrollo todavía no ha logrado un encaje pleno en las enseñanzas universitarias.

Palabras clave: educación para el desarrollo, Universidad, formación, cooperación para el desarrollo, valores.

${ }^{a}$ Departamento de Sociología y Antropología Social. Universidad de Valencia.

${ }^{\mathrm{b}}$ Departamento de Trabajo Social. Universidad de Valencia

${ }^{*}$ Correspondencia: Universidad de Valencia. Campus Tarongers. Avenida de Tarongers 4-B. 46021 Valencia. España.

E-mail: joan.lacomba@uv.es 
Abstract: Education for development has progressively become more popular in Spanish universities in parallel with the extension of cooperation for development between them. Education for development has been seen as an opportunity to promote the university's social commitment and foster values of solidarity and justice through university education. Education for development has highlighted the educational component of the university, often subsumed by the desire for training. However, the scope of education for development and, specially, its mainstreaming in university study programs remain extremely limited. The objective of this article is to show what development education is and what role it can play in the context of university education. To this purpose we draw on to the analysis of secondary sources, by reviewing both specialized bibliography and gray literature. Our main conclusion is that education for development has not yet achieved a full adaptation to university education.

Keywords: education for development, university, training, cooperation for development, values.

\section{INTRODUCCIÓN}

Este artículo aborda el sentido que la educación para el desarrollo adquiere en el marco de la actual enseñanza universitaria. En un mundo con enormes retos sociales -las desigualdades persistentes en el desarrollo entre países y poblaciones dentro de estos, los efectos del cambio climático o los intensos movimientos migratorios- la Universidad no puede permanecer insensible a ellos. La institución universitaria surge como un espacio de difusión y universalización del conocimiento y adquiere una creciente responsabilidad en la mejora del mundo. El compromiso social de la Universidad se ha convertido también en parte insoslayable de esta. No obstante, a lo largo del tiempo se ha vivido la tensión entre dos modelos o dos formas de entender el papel de la institución universitaria: la Universidad como espacio educativo (en el sentido humanístico del término) y la Universidad como espacio formativo (en el sentido de preparación específica para el ejercicio de una actividad profesional en el mercado laboral).

Estas dos visiones en torno al papel de la Universidad tienen tras sí sendas maneras de considerar la educación: la educación como capacidad en sí misma y como base para la ampliación de otras capacidades humanas -en el sentido que Amartya Sen (2000) atribuye a estas-, frente a la educación como formación de capital humano. Desde la primera perspectiva, tal como explica Cejudo sobre la base del trabajo de Sen, "la finalidad de la educación puede interpretarse como el aumento del conjunto de capacidad de las personas a 
través de la adquisición de capacidades para funcionar" (2006, p. 373). La educación, entendida así como proceso que favorece una mayor libertad, se convierte en una contribución fundamental para el desarrollo humano. El resultado de la educación no puede ser el hecho en sí de "estar formado", sino que su principal logro debería ser ampliar las capacidades que permiten alcanzar una mayor libertad en beneficio del desarrollo. La dimensión emancipatoria de la educación, mediante la posibilidad de experimentar elecciones autónomas, constituye, pues, la esencia de esta.

En el contexto de la reforma de las enseñanzas universitarias iniciado con el Proceso de Bolonia, se privilegió una visión instrumental de la educación y se otorgó un importante peso al desarrollo de competencias centradas sobre todo en las demandas del mercado laboral, pero la necesidad de otro tipo de competencias sociales sigue estando ahí. Pese a lo avanzado, hay que seguir recordando que "el aprendizaje no se agota en la mera adquisición de conocimientos, sino que incluye la adquisición de hábitos, valores, destrezas, habilidades, actitudes y normas de conducta" (De la Torre, 1991, p. 177). En realidad, no es posible enseñar sin valores, porque siempre se enseña a alguien para algo. Como señala Fernández Gracia,

... hay una dimensión axiológica en toda ciencia, en toda rama del saber. De no asumir este hecho, estamos dirigiendo-deseducando en otro sentido. La economía, el derecho, la estadística... por citar la más aparentemente ajenas a ello, deben incorporar una cierta visión de las actitudes que mueven al hombre, deben pues enseñar, en la medida en que su discurso lo permita, a criticar, a discernir, a vivir los valores (1996, p. 28).

No hablemos ya de los estudios humanísticos, educativos y las ciencias sociales, donde esta aparente obviedad se convierte en su esencia. Sin embargo, siguen existiendo importantes resistencias al reconocimiento de la educación en valores como parte de la educación universitaria.

La educación para el desarrollo, en sus diferentes conceptualizaciones y formulaciones, ofrece la posibilidad de avanzar en este camino. Sin embargo, como sostiene Fueyo "la ED ha tenido una tardía y débil implantación en la Universidad y se ha caracterizado por la diversidad de prácticas derivadas de la gran heterogeneidad, complejidad y diversidad de intereses en el seno de las instituciones universitarias" (2012, p. 47). 


\section{Por unA PROpuesta EDUCATIVA GLOBAL y huMANIZADORA}

Hace ya mucho tiempo que la Unesco destacó que las universidades agrupan el conjunto de las funciones tradicionales asociadas al progreso y la transmisión del saber: investigación, innovación, enseñanza y formación, educación permanente. Señaló, además, que a esas funciones se puede agregar otra que desde hace algunos años cobra cada vez más importancia: la cooperación internacional. Por ello, concluía la Unesco, "las instituciones de enseñanza superior están admirablemente situadas para sacar partido de la mundialización a fin de colmar el déficit de saber y enriquecer el diálogo entre los pueblos y entre las culturas" (Unesco, 1996, p. 149).

De acuerdo con la Unesco, la educación tiene como una de sus tareas fundamentales la de ayudar a transformar una interdependencia de hecho en solidaridad deseada. Desde esta perspectiva, la educación

... debe lograr que cada persona pueda comprenderse a sí misma y a las demás mediante un mejor conocimiento del mundo. Para que uno pueda comprender la complejidad creciente de los fenómenos mundiales y dominar el sentimiento de incertidumbre que suscita, en primer lugar, debe adquirir un conjunto de conocimientos y luego aprender a relativizar los hechos y a tener espíritu crítico frente a las corrientes de información (Unesco, 1996, p. 51).

Esta labor transformadora ha venido siendo reivindicada por la propia Unesco desde su creación, instando en la recomendación de 1974 a los Estados y a las organizaciones que desarrollan una labor educativa a considerar la educación como un medio para solucionar los problemas fundamentales que condicionan la supervivencia y el bienestar de la humanidad (desigualdad, injusticia, relaciones internacionales basadas en el uso de la fuerza) y las medidas de cooperación que puedan facilitar su solución.

Esta forma de entender la educación se vincula con la filosofía del aprender a aprender popularizada por Jacques Delors en el libro La educación encierra un tesoro. Informe a la Unesco de la Comisión Internacional sobre la educación para el siglo XXI (Unesco, 1996), un programa que combina las necesarias reorientaciones pedagógicas con el conocimiento profundo de la realidad actual en la que vivimos, con el objetivo de formar ciudadanos críticos, autónomos, tolerantes y solidarios. 
La filosofía educativa de la Unesco se sostiene en cuatro pilares: aprender a conocer, aprender a hacer, aprender a vivir juntos y aprender a ser.

En primer lugar, aprender a conocer significa que hay combinar una cultura general suficientemente amplia con la posibilidad de profundizar los conocimientos en un pequeño número de materias.

En segundo lugar, aprender a hacer permite adquirir no solo una calificación profesional sino una competencia que capacite para hacer frente a gran número de situaciones y a trabajar en equipo.

En tercer lugar, aprender a vivir juntos pasa por desarrollar la comprensión del otro y la percepción de las formas de interdependencia -realizar proyectos comunes y prepararse para tratar los conflictos- respetando los valores de pluralismo, comprensión mutua y paz.

En cuarto lugar, aprender a ser implica la propia personalidad, potenciando las condiciones de obrar con creciente capacidad de autonomía, de juicio y de responsabilidad personal (Unesco, 1996, p. 109).

Los cuatro pilares de la educación enunciados están cimentados sobre una misma base, que consiste en educar para aprender a aprender. Esta premisa supone, entre otras cosas, el reconocimiento de que la esencia del proceso pedagógico es la fuerte relación que se establece entre el docente y el alumno. De manera, que

... el trabajo del docente no consiste solo en transmitir conocimientos, sino en presentarlos en forma de problemática, situándolos en un contexto y poniendo los problemas en perspectiva, de manera que el alumno pueda establecer el nexo entre su solución y otros interrogantes de mayor alcance. La relación pedagógica trata de lograr el pleno desarrollo de la personalidad del alumno respetando su autonomía (Unesco, 1999, p. 166).

En su contribución al libro La educación encierra un tesoro, Bob Geremek abunda en esta idea y señala que el concepto de aprender a ser

... remite al respeto primordial de la persona humana en las relaciones sociales y políticas, en la relación entre el hombre y la naturaleza, en la confrontación de las civilizaciones y las economías (...) Tratando de comprender lo real -el hombre y el mundo- hay que aprender las interdependencias que crean la nece- 
sidad de solidaridades. Solidaridades que no son cuestión de buenas intenciones, sino que resultan de las limitaciones del tiempo actual (1996, p. 253).

En esta misma línea, Rafael Yus habla de la necesidad de una educación global desde unos principios mundialistas y sobre la base de una ética de mínimos, basada en una concepción más holística. Sostiene Yus que

... la toma de conciencia de los problemas globales ha puesto de manifiesto la insuficiencia y miopía de los planteamientos localistas. A una escala global, se difuminan las barreras entre los temas transversales, de tal suerte que todos aparecen íntimamente interdependientes, ofreciéndonos un mayor poder explicativo de los problemas que actualmente aquejan a la humanidad y un mejor enfoque educativo para una ciudadanía del mundo (1996, p. 11).

Sin embargo, así como en otros niveles educativos se viene desarrollando un amplio debate y trabajo sobre la introducción de nuevas dimensiones curriculares, de acuerdo con las tendencias mundiales apuntadas, en el nivel universitario se ha avanzado de manera más lenta. La cuestión de los temas transversales en la Universidad (educación para la solidaridad, la cooperación, el desarrollo o la diversidad), sigue ocupando aún un lugar marginal en el currículum universitario. Ese humanismo global del que habla Mel Gurtov -entendido como una serie de valores y normas humanos que nos permite examinar las políticas, las ideologías, las fuerzas sociales y las instituciones desde el punto de vista de las necesidades e intereses del planeta, considerado como una comunidad humana y como un sistema ecológico (Gurtov, 1990, p. 20)- todavía no ha acabado de arraigar en las aulas universitarias.

\section{3. ¿QUÉ ES LA EDUCACIÓN PARA EL DESARROLLO?}

De acuerdo con Manuela Mesa, la Asociación Nacional de Centros de Educación para el Desarrollo (NADEC) del Reino Unido definió esta, en los años ochenta, como

... la práctica educativa que pretende hacernos capaces de comprender los vínculos entre nuestra propias vidas y las de otros pueblos a lo largo del mundo; 
incrementar la comprensión de las fuerzas económicas, sociales, políticas y ambientales que dan forma a nuestras vidas; desarrollar las actitudes, habilidades y valores que permiten trabajar conjuntamente en favor del cambio y tomar control de sus propias vidas; y lograr un mundo más justo, en el que el poder y los recursos sean compartidos equitativamente por todos (Mesa, 1994, p. 57).

Esta definición englobaría las cuestiones norte-sur, pero también los problemas ambientales y la perspectiva multicultural y de igualdad de oportunidades, a través de la comprensión y empatía con las culturas, valores y formas de vida de las personas de nuestro entorno local e internacional.

En cuanto al Ministerio de Cooperación Económica y Desarrollo alemán, este estableció en 1992 que el objetivo de la educación para el desarrollo es

... aumentar la comprensión de la población sobre las causas estructurales del desarrollo-subdesarrollo y el conjunto global de problemas interrelacionados con ello, mostrando la urgencia de los problemas de desarrollo y las repercusiones directas que tiene en la sociedad, promoviendo la solidaridad y las relaciones con las ONGs del Sur, incluyendo además la cuestión multicultural (Mesa, 1994, p. 58)

En España, Rafael Grasa escribía en 1990 que la educación para el desarrollo debe entenderse como una práctica educativa orientada hacia el cambio social y como correlato del conjunto de aspiraciones y prácticas de los actores comprometidos con otro modelo de desarrollo y otro tipo de relaciones. De modo que nada puede entenderse en temas de cooperación y desarrollo sin aprehender el contexto general de las relaciones internacionales (1990, p. 97). Partiendo de esta premisa, la educación para el desarrollo no solo depende de sus propios objetivos, estrategias y métodos, sino también del impacto real que provoquen los cambios del sistema internacional y de las relaciones Norte-Sur. Son producto de ello el debate sobre la reconceptualización del desarrollo y sobre los objetivos, estrategias, instrumentos y finalidades de la propia cooperación.

Por su parte, Fernando Marhuenda (1994) afirmaba que la educación para el desarrollo, desde el punto de vista de la educación formal, es entendida como la inserción de los temas de las desigualdades, injusticias, desequilibrios norte-sur, paz, solidaridad y desarrollo en el contexto educativo, con el 
objetivo de contribuir a la sensibilización, formación y concienciación de los/ las estudiantes de los países occidentales.

Por tanto, la educación para el desarrollo incluye objetivos cognitivos (dirigidos a un mayor conocimiento de la realidad norte-sur), objetivos aptitudinales (aquellas capacidades necesarias para entender la realidad norte-sur desde una perspectiva global y desde nuestro entorno local) y objetivos actitudinales que potenciar y estimular (como actitudes responsables ante sí mismos, los demás y nuestro entorno, la solidaridad con otros pueblos y personas, la tolerancia y el respeto hacia otras formas de vida, la cooperación como forma de trabajo, el respeto por los derechos humanos, la participación y comunicación, la creatividad, la curiosidad, la coeducación e igualdad entre los sexos, actitudes abiertas a nuevos planteamientos e ideas que buscan una mayor justicia social).

En cuanto a los argumentos a favor de una educación para el desarrollo, estos pueden ser muy diversos. Anna Ros (1995) trató de sintetizarlos en doce dimensiones: 1) Los derechos y libertades están por encima de las fronteras del Estados nación y tienen su contrapartida en los deberes de las personas y los grupos. 2) Se reconoce la creciente interdependencia entre los pueblos. 3) Hay un reconocimiento internacional de la diversidad cultural y su riqueza. 4) Se necesita aprender valores y culturas no occidentales. 5) Los habitantes del presente somos los guardianes del entorno. 6) Se necesita resolver pacíficamente los conflictos humanos. 7) Se necesita una educación para la comunidad. 8) Se deben enfatizar las semejanzas entre la humanidad. 9) Se deben situar los objetivos de la educación para el desarrollo como los objetivos básicos de todo sistema educativo democrático. 10) La educación para el desarrollo es una forma de mirar el mundo y sus problemas que nos sirve para poder entenderlo y mejorarlo. 11) Es un proceso de descubrimiento y conocimiento permanente. 12) La educación para el desarrollo está al servicio de la emancipación, de la liberación de la alienación y la opresión por medio del aprendizaje.

\section{UNA PEDAGOGÍA DE LA EDUCACIÓN PARA EL DESARROLLO}

Pero la educación para el desarrollo no solo permite conocer mejor el mundo en el que vivimos, sino que también se asienta en unas sólidas bases pedagógicas, coherentes con los valores que la recorren. Argibay y Celorio nos recuerdan que 
... la dimensión pedagógica de la ED está dentro de su propio enunciado que nos habla de educar para lograr algo llamado desarrollo. Presupone un acto volitivo, estructurado, basado en técnicas de construcción del saber y métodos de formación que conseguirán transformar a las personas en promotores del cambio social hacia el Desarrollo (2015, p. 75).

Diversos autores nos recuerdan que en la educación para el desarrollo debe existir una alta coherencia entre fines y medios, entre métodos, discurso y contenidos de la propuesta formativa. La educación para el desarrollo, que propugna valores solidarios, cooperativos, críticos, de participación, no puede llevarse a cabo mediante métodos tradicionales, jerárquicos, individualistas, de transmisión vertical del conocimiento. En la educación para el desarrollo no puede separarse texto y contexto, método y contenido. Sin embargo, no siempre ocurre así. En muchos casos la dimensión metodológica es considerada, injustamente, un aspecto secundario respecto al contenido. Por ello, Manuela Mesa insiste en que en la enseñanza

... a menudo se siguen utilizando esquemas tradicionales de aprendizaje -con una concepción del conocimiento muy compartimentalizada- que hacen difícil la introducción de enfoques globales y de metodologías participativas para trabajar con el grupo. La educación para el desarrollo, sin embargo, se caracteriza por su dimensión globalizadora, abordando distintos aspectos de la realidad y analizando las interrelaciones que existen desde una perspectiva local-global. Es además interdisciplinaria, porque no se circunscribe a una sola asignatura y propone un trabajo entre las distintas materias (Mesa, 1994, p. 195).

La educación para el desarrollo entronca con los postulados pedagógicos de Paulo Freire y su concepción problematizadora de la educación, que implica un acto permanente de desvelamiento de la realidad y la generación de temas a partir del grupo. Los elementos fundamentales de esta metodología se basan en la reciprocidad de conciencias: no hay profesor/a (en el sentido clásico), sino un coordinador/a que tiene por función dar las informaciones solicitadas por los respectivos participantes y propiciar las condiciones favorables a la dinámica del grupo.

Desde esta perspectiva, los rasgos metodológicos característicos de la educación para el desarrollo serían: el grupo como proceso, el protagonismo del 
grupo (creando un clima de grupo de formación), un enfoque integral, el descubrimiento paulatino de las necesidades de formación, una formación orientada a la acción, una formación abierta a la realidad y al entorno, la evaluación como parte del proceso de aprendizaje (Pérez Serrano, 1997, p. 113).

Por su parte, Pedro Sáez (1993) señala como principales orientaciones metodológicas en la educación para el desarrollo, las siguientes:

1. El análisis de las percepciones: conocer la imagen que se tiene sobre los países del sur para cuestionar los prejuicios y estereotipos que bloquean el aprendizaje y el respeto hacia otros pueblos y culturas, revisando nuestro modelo de desarrollo.

2. El análisis de las interrelaciones entre lo local y lo global: destacar las interconexiones e interrelaciones entre lo que ocurre aquí y allí, para traer el tercer mundo a casa. Concienciar de que vivimos en un solo mundo, interdependiente en lo económico, político, social y cultural.

El propio Pedro Sáez reclama la elaboración de una serie de claves pedagógicas que permitan llevar la "didáctica del Sur" a los espacios educativos. Esas claves pedagógicas se articulan en torno a tres conceptos básicos: conflicto, globalización y solidaridad. La noción de conflicto abre las puertas a una lectura crítica, dinámica y multidisciplinar de la realidad en la que se inscribe la problemática. La noción de globalización se convierte en un recurso metodológico que sistematiza una manera de comprender los fenómenos interrelacionados con el eje norte-sur. La noción de solidaridad permite construir a su alrededor actitudes personales y proyectos sociales cooperativos y emancipadores.

En cuanto a la didáctica de la globalización, esta tiene como objetivo generar una visión interrelacionada de la realidad humana para promover una intervención activa y crítica sobre esta. Se pone así de manifiesto la interdependencia de los conflictos derivados de las relaciones norte-sur y la necesidad de enfrentarse con la complejidad de esa relación.

Respecto a la didáctica de la solidaridad, se entiende como el intento de situar esta como núcleo central y referente ético del proceso de aprendizaje, a través de una serie de actitudes abiertas a la participación social, el diálogo cooperativo y la imaginación creadora. 
También otros autores han coincidido en la importancia que la solidaridad ocupa dentro de la educación para el desarrollo, y que puede condensarse en la idea expresada por Gloria Pérez Serrano; en cuanto que

... la solidaridad se articula en modos históricos de cooperación e interdependencia, se nutre de actitudes y acciones, de comprensión y respeto de los derechos y libertades de las personas y de los grupos humanos. Todo esto quiere decir que hay que aprender a asumir y vivir unos valores nuevos, una ética humanista de solidaridad que presupone alcanzar una visión nueva de la vida, de los seres humanos, de sus relaciones y del mismo planeta en que viven. La palabra solidaridad sugiere también compromiso, acción, no sólo declaración de intenciones. La solidaridad no es, pues, solamente la fundamentación y la motivación, ni el objetivo final a conseguir, sino que es también el estilo de la cooperación, de la comprensión, del civismo e interdependencia de la educación (1997, p. 115).

Por ello Gloria Pérez Serrano dice, en su libro Cómo educar para la democracia, que la educación para el desarrollo se fundamenta en la interdependencia y la solidaridad entre los pueblos. Solidaridad a través del estudio y valoración de los desequilibrios socioculturales y económicos dentro y entre los estados, haciendo hincapié en los países más empobrecidos. Así, en tanto que somos cada vez más sensibles a los problemas que se presentan en otros países, debido a la información detallada y puntual de los hechos y situaciones que nos hacen sentirnos próximos, esta información nos lleva a la creación de una conciencia planetaria que nos impulsa y vincula con los problemas de todos (Pérez Serrano, 1997, p. 111).

La educación para el desarrollo parte, pues, de la concepción de la educación como un ámbito de discusión y crítica cultural, así como un espacio de formación en actitudes y valores encaminados al compromiso personal y colectivo con los principales problemas actuales. Sus objetivos tratan de poner de manifiesto no solo la existencia de desigualdades a nivel local y global, sino también mostrar las posibilidades de transformación de la realidad.

Al mismo tiempo, la educación para el desarrollo mantiene vínculos con la educación para la paz, la educación internacional, la educación para la cooperación, la educación para la ciudadanía, la educación intercultural o la educación global, así como con la educación en los derechos humanos, la educación 
antirracista, la educación ambiental, la educación para la salud o la educación para el consumo.

Todos estos ámbitos con los que se conecta y qué conecta la educación para el desarrollo han hecho que, más recientemente, se pase a hablar de forma más amplia de una educación para la ciudadanía global (ECG). La ECG se concibe como "un proceso de aprendizaje dinámico abierto a los cambios que se producen en el contexto internacional, capaz de incorporar nuevas visiones y enfoques en el ámbito global y local" (Mesa, 2019, p. 7). Algo similar ocurre con la educación para el desarrollo sostenible (EDS), una denominación que ha extendido la Unesco en los últimos años para poner el énfasis en la necesidad de sensibilizar en la protección del medio ambiente y el equilibrio con las actividades económicas. La EDS se entiende como un aprendizaje interactivo alrededor de cuestiones como el cambio climático, el consumo o la producción sostenible con un objetivo de transformación social (Unesco, 2016).

Estas nuevas formulaciones tienden a desplazar la más clásica de educación para el desarrollo.

\section{LAS OTRAS EDUCACIONES}

La educación para el desarrollo ha ido tomando cuerpo en contacto con otros modos alternativos de educar y entender el mundo, con los que se ha retroalimentado y comparte numerosos elementos. Así, por ejemplo, en Bélgica la coordinadora flamenca de ONG para el desarrollo (NCOS) hablaba en los años ochenta de la educación mundial como la educación que enseña a los jóvenes a vivir en su comunidad local y en su comunidad mundial. La educación mundial requeriría de un mayor conocimiento de la interdependencia global, de su realidad más cercana, una relativización de nuestros propios valores, y una aproximación positiva a los valores de otras personas, culturas y pueblos.

Manuela Mesa apuntaba que esta amplia definición permite abarcar tanto los contenidos de la educación para el desarrollo en sentido estricto -relaciones norte/sur, subdesarrollo y cooperación- como en sentido amplio -medio ambiente global, derechos humanos, paz, armamentismo, racismo y educación multicultural-. Y, al igual que la educación para el desarrollo, también alude al proceso educativo. En este sentido, la educación mundial no solo se refiere 
a la transmisión de contenidos, sino también a la formación de habilidades, actitudes y valores. El punto central es la concienciación acerca de las causas reales de muchos de nuestros problemas concretos, que están relacionados con problemas globales que solo pueden ser resueltos tanto a partir de la acción local como de cambios estructurales de alcance mundial (Mesa, 1994, p. 48).

Muy cercana a la educación mundial se encuentra también la educación para la paz, entendida como

... un proceso educativo, continuo y permanente, fundamentado en los dos conceptos definidores, la concepción positiva de paz y la perspectiva creativa del conflicto, y que a través de la aplicación de métodos problematizantes pretende desarrollar un nuevo tipo de cultura, la cultura de la paz, que ayude a las personas a desvelar críticamente la realidad, desigual, violenta, compleja y conflictiva, para poder situarse ante ella y actuar en consecuencia (Jares, 1995, p. 6).

La educación para la paz trata de potenciar la paz positiva, considerada esta "como la ausencia de violencia tanto directa como estructural, y como la forma de construir relaciones más justas e igualitarias en y entre las sociedades" (Mesa, 1994, p. 22).

Para Gloria Pérez Serrano la preocupación por la paz y la convivencia internacional responde a una creciente toma de conciencia mundial ante los grandes problemas a los que se enfrenta la humanidad. Y considera

... que se debe fomentar, en la escuela y en los centros universitarios, la educación para la paz, pues este valor forma un entramado con otros valores ligados entre sí que se exigen unos a otros. Así, la educación para la paz exige formar el espíritu crítico, la capacidad de dialogar, la veracidad, la coherencia de mediosfines y la justicia. La educación para la paz no es compatible con el individualismo, la insolidaridad, la competitividad, la discriminación, etc. (1997, p. 97).

La educación para la paz se sostendría en principios como: el fomento del diálogo, el aprendizaje cooperativo, el desarrollo de la autoestima y el fomento de actitudes democráticas.

Por su parte, Jean Paul Lederach afirma que la educación para la paz tiene una doble finalidad: primero, la investigación de los obstáculos y las causas que nos impiden lograr una condición de elevada justicia y reducida violencia; y, 
segundo, el desarrollo de conocimientos, valores y capacidades para emprender y edificar el proceso que lleva hacia la más plena realización de la paz (1994, p. 35). Por tanto, la educación para la paz no puede ser una actividad meramente teórica, también debe motivar comportamientos activos en favor de la paz.

Junto a la necesidad de educar para la comprensión internacional y la paz, situamos también la necesidad de educar para la diversidad, en un mundo con graves problemas de intolerancia y débil diálogo cultural. Toma sentido así la educación intercultural en un contexto de toma de conciencia del aumento del grado de diversidad y la configuración de sociedades cada vez más multiculturales.

Como dice Manuela Mesa, el fenómeno migratorio, acentuado por la crisis del desarrollo, y el hecho de que a menudo los inmigrantes padecen situaciones de marginación que los convierten en un sur dentro del norte han acentuado la importancia de la educación multicultural en Europa, y las actividades de sensibilización y educación para el desarrollo se encuentran desde mediados de los ochenta cada vez más impregnadas de contenidos y metodologías referidas a las interrelaciones de la globalización económica, la crisis del desarrollo, los flujos migratorios, su impacto en los países receptores, la situación de los inmigrantes, la xenofobia y el racismo (Mesa, 1994, p. 26).

Es cierto que asistimos a la configuración de sociedades cada vez más complejas, más heterogéneas y diversas como resultado de la creciente interdependencia mundial y el consecuente aumento de la movilidad de las personas en busca de nuevos modos de vida y una mejora de sus condiciones socioeconómicas. Son esos cambios, y los conflictos generados por esas nuevas realidades, los que nos han hecho tomar conciencia de la importancia de desarrollar vías de encuentro y diálogo con los otros, de manera muy particular en el terreno de los movimientos migratorios.

El aumento de los efectivos extranjeros ha ido acompañado igualmente de modificaciones en sus características y de una mayor visibilidad de los colectivos inmigrantes. Las modificaciones afectan a las características sociodemográficas y la composición nacional y religiosa de la población extranjera, en cuanto a la atenuación de las especificidades sociodemográficas y una diversificación de los orígenes nacionales marcada por el aumento de las nacionalidades extraeuropeas. En cuanto a la dimensión religiosa-cultural sigue 
siendo un elemento ambiguo y confuso en su valoración como factor positivo o negativo de integración de la población inmigrante.

En todo caso, el debate actual acerca de la presencia inmigrante se desarrolla a dos niveles: a nivel cultural, se polemiza sobre el riesgo de disminución de la cohesión de la sociedad y una crisis identitaria, al tiempo que se extiende la idea de que la exclusión de los inmigrantes podría desembocar en la constitución de minorías. A nivel económico, existe un extendido temor a una competencia laboral por el empleo y los salarios. Son todos ellos extremos que matizar, analizar, y sobre los que cabe profundizar para contrarrestar los efectos socioculturales de una visión negativa de la inmigración.

En el caso del Estado español, y junto a su característica diversidad nacional y lingüística, un fenómeno en aumento en la última década ha sido el que se conoce como "nueva inmigración", en la medida en que asistimos a la presencia cada vez más visible de una inmigración considerablemente diversificada en cuanto a sus procedencias geográficas y culturales, con un peso cada vez mayor de personas originarias de los países del tercer mundo.

Ese hecho ha puesto de actualidad la necesidad de elaborar nuevos enfoques que permitan abordar el fenómeno migratorio y diseñar nuevas líneas de intervención social con el fin de facilitar la inserción de los nuevos colectivos. Igualmente se plantea la urgencia de poner en marcha medidas que actúen como freno a la exclusión y que, al mismo tiempo, contrarresten actitudes y acciones de carácter racista y xenófobo.

Todo ello no excluye al mismo tiempo el reconocimiento de que la existencia de una sociedad multicultural implica necesariamente la presencia de conflictos. De la misma manera los conflictos derivados de la multiculturalidad pueden ser considerados como positivos para la sociedad. Como indica el Colectivo Amani, desde una visión positiva de los conflictos el planteamiento pedagógico de la educación intercultural no pasa por eludir el conflicto, sino por afrontarlo directamente y sacarlo a la luz cuando está latente (Colectivo Amani, 1994, p. 11).

La educación intercultural se plantea, desde esta perspectiva, como el proceso educativo que trata de responder al conflicto planteado por la realidad multicultural. Los conflictos interculturales se producen entre personas de diferentes culturas, pero esta pertenencia diferencial no es la causa que los 
provoca, sino que estamos ante conflictos fundamentalmente sociales. De hecho, los conflictos son inherentes a la coexistencia de personas de diferentes culturas en una misma sociedad y nos permiten crecer dialécticamente.

Desde este punto de vista, una propuesta educativa intercultural debe tener como objetivos los siguientes:

1. Conocer y modificar los estereotipos y los prejuicios entre mayorías y minorías.

2. Favorecer el conocimiento y la valoración positiva y crítica de las culturas mayoritarias y minoritarias.

3. Propiciar una toma de conciencia sobre la necesidad de un mundo más justo e igualitario.

4. Promover actitudes, conductas y cambios sociales positivos que eviten la discriminación y favorezcan las relaciones positivas, posibilitando el desarrollo específico de las culturas minoritarias y la mejora de sus condiciones de vida.

En suma, la educación intercultural pretende facilitar el conocimiento, la comprensión, afirmación, empatía y desarrollo de la diversidad cultural, religiosa, étnica y de otros tipos de identidad -incluyendo la propia-, superando actitudes y prejuicios negativos. La educación debe preparar a las personas para una vida en una sociedad de religiones, culturas, costumbres y estilos de vida variados, cada uno diferente pero interdependientes, potenciando actitudes y modos de comportamiento adecuados para erradicar los prejuicios raciales (Mesa, 1994, p. 25). Pero también debe prepararlas para el trabajo profesional en contextos donde es fundamental abordar el tratamiento de la diversidad cultural, donde "la perspectiva pluricultural inherente a un trabajo social con usuarios de diferentes orígenes étnicos y religiosos, nos lleva a modificar no solo la definición de problemáticas sino también las concepciones técnicas de acercamiento y tratamiento de la clientela" (Hill, 1992, p. 45).

Las nuevas generaciones han entrado en contacto con otros jóvenes inmigrantes o hijos de inmigrantes (las llamadas segundas generaciones) en las aulas de las escuelas, los institutos y, más recientemente, en las universidades. No obstante, pese a este contacto cotidiano, y seguramente una mayor naturalidad de los jóvenes en la aceptación de la diversidad, los prejuicios tampoco se han diluido automáticamente, lo que obliga a seguir trabajando para sentar las bases 
de una convivencia razonable. El trabajo en las aulas y el uso de nuevos materiales pedagógicos quizás no logren resolver todos los problemas existentes, pero constituyen una herramienta fundamental y obligada para promover un mejor encaje de las diferencias.

\section{LA EDUCACIÓN PARA EL DESARROLLO EN LA UNIVERSIDAD}

La educación para el desarrollo tiene sus primeros antecedentes en el ámbito educativo en el impulso dado por la Unesco en los años setenta a la educación para la comprensión internacional (Classen-Bauer, 1979; Richardson, 1979). El énfasis de la Unesco se vio reflejado en trabajos como el de Lester Brown, en uno de cuyos artículos sostenía que

la comprensión internacional, que antaño fue una virtud humanitaria, es hoy una necesidad dramática y urgente: la interdependencia de las diferentes regiones y países del mundo y la tensión ejercida sobre los recursos limitados son tales que sin una comprensión que conduzca a una cooperación, se producirá el desastre (1979, p. 168).

Esta idea comenzó a hacerse presente a partir de los años ochenta en los programas educativos de países como Gran Bretaña y Alemania, y tuvo sus reflejos en los primeros trabajos españoles sobre la educación para el desarrollo en la década de los noventa. Sin embargo, la educación para el desarrollo no se hace visible en las universidades españolas hasta comienzos del siglo XXI. A partir de ahí, la educación para el desarrollo ha ido ganando peso en las universidades de la mano, sobre todo, de la cooperación para el desarrollo.

En las dos últimas décadas las universidades se han implicado en acciones de desarrollo en los países del sur y se han convertido en importantes actores del propio sistema de cooperación para el desarrollo (según el Observatorio de la Cooperación Universitaria al Desarrollo, OCUD, la Ayuda Oficial al Desarrollo universitaria supuso en 2015 el 0,57 \% del total de la AOD española y un $1,52 \%$ de la AOD bilateral).

Esta creciente participación ha dado lugar a la institucionalización de la cooperación universitaria al desarrollo (CUD). La propia Conferencia de 
Rectores de las Universidades Españolas (CRUE) definió en 2015 el código de conducta de las Universidades en materia de cooperación al desarrollo, un texto creado y aprobado en el seno de la Comisión de Internacionalización y Cooperación.

En el artículo 9 de dicho código se entiende la cooperación universitaria al desarrollo "como el conjunto de actividades llevadas a cabo por la comunidad universitaria y orientadas a la transformación social en los países más desfavorecidos, en pro de la paz, la equidad, el desarrollo humano y la sostenibilidad medioambiental en el mundo, transformación en la que el fortalecimiento institucional y académico tienen un importante papel". La cooperación universitaria al desarrollo incluye acciones de formación (teórica y práctica), acciones de investigación, cooperación interuniversitaria encaminada al fortalecimiento institucional, acciones de difusión, sensibilización, movilización y educación para el desarrollo, programas y proyectos de acción sobre el terreno, así como promoción, gestión y coordinación de políticas y programas CUD.

Además, toda esta diversidad de acciones se relaciona con el compromiso de las universidades adquirido en el cumplimiento de los objetivos de desarrollo sostenible de Naciones Unidas promovidos por la Agenda 2030, en especial cuando se habla del Objetivo 4 (garantizar una educación inclusiva, equitativa y de calidad y promover oportunidades de aprendizaje durante toda la vida para todos), pero también del resto de objetivos a los que el papel de la Universidad puede contribuir.

En este sentido, la educación para el desarrollo en el seno de la Universidad también contribuye a impulsar las nuevas concepciones del desarrollo, como los paradigmas del desarrollo humano o el desarrollo sostenible. La noción de desarrollo humano - popularizada por el Programa de Naciones Unidas para el Desarrollo- descansa en las aportaciones de Amartya Sen "respecto a la necesidad de contemplar al ser humano como protagonista y destinatario del desarrollo, como fin en sí mismo, en lugar de ser considerado como medio para el logro de otros fines" (Unceta, 1996, p. 71). En cuanto a la noción de desarrollo sostenible, esta "viene a enfatizar la necesidad de hacer compatible el desarrollo con la utilización racional de los recursos, y con la vida de las futuras generaciones" (Unceta, 1996, p. 72). Desarrollo humano y desarrollo sostenible forman parte cada vez más común del lenguaje docente y de la investigación universitaria. Ambos términos apuntan a una modificación en 
la forma de entender el desarrollo y a un cambio en las actitudes frente a los problemas del mundo actual.

Sin embargo, pese a algunos avances en la incorporación de la educación para el desarrollo en las universidades, en buena parte de los casos los contenidos y las competencias asociados a esta permanecen vinculados a asignaturas optativas o actividades de libre configuración, o se desarrollan en formaciones de posgrado (másteres y cursos de especialización en cooperación para el desarrollo). Precisamente, la concentración de la cooperación al desarrollo -y por extensión de la educación para el desarrollo- en la formación de posgrado ha limitado la transversalización de esta última en el conjunto de los estudios universitarios. La incorporación transversal de la educación para el desarrollo en los grados universitarios resulta muy excepcional (Fueyo, 2012), en especial cuando se trata de titulaciones alejadas de las humanidades y las ciencias sociales, como las enseñanzas técnicas. La cuestión de la transversalidad sigue en realidad sin resolverse, y la educación para el desarrollo sigue siendo considerada un contenido más que un enfoque educativo.

\section{Conclusiones}

La Universidad vive la tensión entre su dimensión educativa y formativa, acrecentada con las reformas iniciadas con la convergencia del espacio europeo. La disyuntiva entre la formación de profesionales con competencias técnicas y la educación de ciudadanos preparados profesional y humanamente pone de relieve el papel de la educación para el desarrollo como estrategia educativa. La educación para el desarrollo apela a la necesidad de ir más allá de la capacitación para el ejercicio de una profesión, potenciando una serie de valores y actitudes clave para abordar los problemas del mundo cada vez más complejo y globalizado en el que vivimos. La educación para el desarrollo, entendida como una herramienta de transformación social, ha de integrar valores de solidaridad y potenciar el compromiso social.

La Universidad constituye un espacio fundamental para implementar el enfoque de la educación para el desarrollo. Los jóvenes, y en especial los jóvenes universitarios, representan la población sobre la que desarrollar nuevos 
valores y actitudes ante los principales problemas del mundo actual (pobreza, desigualdades, subdesarrollo, cambio climático o racismo). Precisamente, los jóvenes han mostrado en los últimos tiempos una importante predisposición a implicarse en la cooperación para el desarrollo en los países del sur, o involucrarse en todo tipo de voluntariados con personas necesitadas de apoyo. Igualmente, los jóvenes son uno de los sectores clave con los que trabajar para lograr una aceptación positiva de la diversidad y la inmigración, no solo porque evidentemente son los adultos del futuro, sino sobre todo porque son ellos quienes más se relacionan con personas de origen inmigrante en los mismos espacios educativos (las escuelas, los institutos y las universidades son hoy en día lugares de una gran diversidad cultural, en donde resulta también más accesible trabajar sobre los prejuicios y estereotipos existentes). Las aulas de la Universidad son un buen espacio en el que educar para la tolerancia, la ciudadanía y la interculturalidad, aunque la labor no debería limitarse únicamente a las aulas, sino que tendría que proyectarse en el espacio social.

La cooperación para el desarrollo, al permitir el conocimiento de otras realidades y la toma de conciencia de la situación de nuestros semejantes, también desempeña un papel esencial en la sensibilización de los jóvenes. No obstante, la educación para el desarrollo trata de ir más allá e incardinarse en el conjunto de las acciones educativas. Aunque la cooperación para el desarrollo sí ha logrado en los últimos años adquirir cierto peso dentro de las universidades, todavía resulta limitada la presencia de la propia educación para el desarrollo, tanto de un modo transversal como de manera específica en materias centradas en su enseñanza. Pese a la importancia de los proyectos y acciones formativas de cooperación universitaria para el desarrollo (CUD), no hay que olvidar que estos no deberían constituir un fin en sí mismo, sino que solo cobran sentido si conseguimos que la Universidad actúe como un agente de transformación social y de las conciencias individuales.

\section{BIBLIOGRAFÍA}

Argibay, M. y Celorio, G. (2005). La Educación para el Desarrollo. Vitoria: Servicio Central de Publicaciones del Gobierno Vasco. 
Brown, L. (1979). Aprendiendo a convivir en un pequeño planeta. Perspectivas IX(2), 168-177.

Cesudo, R. (2006). Desarrollo humano y capacidades. Aplicaciones de la teoría de las capacidades de Amartya Sen a la educación. Revista Española de Pedagogía 234, 365-380.

Celorio, G. y López, A. (coords.) (2012). La Educación para el Desarrollo en la Universidad. Reflexiones en torno a una práctica transformadora. Bilbao: Universidad del País Vasco/Hegoa.

Colectivo Amani (1994). Educación intercultural. Análisis y resolución de conflictos. Madrid: Editorial Popular.

Classen-Bauer, I. (1979). Educación para la comprensión internacional. Perspectivas $I X(2), 186-192$.

CRUE (2015). Código de conducta de las Universidades en materia de Cooperación al Desarrollo. Recuperado de: http://ocud.es/files/doc374/codigoconducta.pdf

De LA TORRE, S. (1991). Un modelo holístico de enseñanza-aprendizaje universitario, en VV. AA., La pedagogia Universitària. Un repte a l'ensenyament superior (pp. 173-179). Barcelona: Universitat de Barcelona.

FERNÁNDEZ, I. (1996). Formación en valores humanos y Trabajo Social. Acciones e Investigaciones Sociales 5, 23-34.

Fueyo, A. (2012). Educación para el Desarrollo como eje transversal en las enseñanzas universitarias, en G. Celorio y A. López (coords.), La Educación para el Desarrollo en la Universidad. Reflexiones en torno a una práctica transformadora (pp. 46-54). Bilbao: Universidad del País Vasco/Hegoa.

GeremeK, B. (1996). Cohesión, solidaridad y exclusión, en Unesco, La educación encierra un tesoro. Informe a la Unesco de la Comisión Internacional sobre la educación para el siglo XXI (pp. 251-256). Madrid: Santillana.

GRASA, Rafael (1990). Aprender la interdependencia: educar para el desarrollo, en Cruz Roja, Educación para el Desarrollo (pp. 97-108). Madrid: Cruz Roja.

Gurtov, M. (1990). Política humanista global. Barcelona: Ediciones PomaresCorredor.

Hill, R. (1992). Nuevos paradigmas en trabajo social. Lo social natural. Madrid: Siglo XXI. 
JARES, X. (1995). Los sustratos teóricos de la educación para la paz. Cuadernos Bakeaz 8.

LEDERACH, J. P. (1984). Educar para la paz. Barcelona: Fontamara.

Marhuenda, F. (1994). La Educación para el Desarrollo en la escuela. Posibilidades e interrogantes. Barcelona: Intermón.

Mesa, M. (ed.) (1994). Educación para el desarrollo y la paz. Experiencias y propuestas en Europa. Madrid: Editorial Popular.

Mesa, M. (2019). La educación para la ciudadanía global y los objetivos de desarrollo sostenible: una agenda para la transformación social. Revista internacional de educación para la justicia social (RIEJS) 8(1), 7-11.

OCUD (2015). Informe sobre la Cooperación Universitaria al Desarrollo 2015. Recuperado de: http://ocud.es/es/pl61/recursos/id2109/informecud-2015.htm

Pérez, G. (1997). ¿Cómo educar para la democracia? Madrid: Editorial Popular.

RichaRdSON, R. (1979). Aprender en un mundo en transformación: criterios y métodos para aplicar en el aula. Perspectivas $I X(2), 193-205$.

Ros, A. (1991). Educación emancipatoria y global. Madrid: Entrepueblos.

SÁEz, P. (1993). El conflicto Norte-Sur. Recursos y estrategias metodológicas. Madrid: CIP.

Sen, A. (2000). Desarrollo y libertad. Barcelona: Planeta.

Unceta, K. (1996). El hambre como fracaso del desarrollo, en B. Sutcliffe (coord.), El incendio frío. Hambre, alimentación y desarrollo (pp. 57-73). Barcelona: Icaria.

UnESCo (1996). La educación encierra un tesoro. Informe a la Unesco de la Comisión Internacional sobre la educación para el siglo XXI. Madrid: Editorial Santillana.

UnESCo (2016). Formando el futuro que queremos: Decenio de las Naciones Unidas de la Educación para el Desarrollo Sostenible (2005-2014). París: Unesco.

Yus, R. (1996). Temas transversales y educación global. Una nueva escuela para un humanismo mundialista. Aula de Innovación Educativa 51, 5-12. 